\title{
Coexistent cerebral metastasis and cavernous malformation
}

W

report the case of a 66 year old man with squamous cell carcinoma of the lung who presented with a seizure. On magnetic resonance imaging (MRI), the contrast enhanced Tl-weighted image showed a $2 \mathrm{~cm}$ lesion suggestive of a cavernous malformation (fig lA). A gradient echo sequence revealed a solitary haemorrhagic focus (fig $1 \mathrm{~B}$ ). The T2-weighted and fluid attenuated images showed an unusual amount of oedema for a cavernous malformation (figs lC, 1D). Despite the imaging, given his history, a metastasis was still suspected.

The patient underwent an image guided stereotactic resection of the lesion. A gross total resection was performed and verified by postoperative imaging. Histological examination revealed haemorrhagic tissue with numerous vessels characteristic of a cavernous malformation (fig lE). Reticulin staining demonstrated scattered foci of small vessels (fig $1 \mathrm{~F}$ ). There were numerous small blue cells with hyperchromatic nuclei consistent with neoplasm (fig IG). Positive staining for keratin strongly suggested squamous cell carcinoma (fig $1 \mathrm{H})$.

While others have reported metastases to vascular malformations, this is the first report of metastasis to a cavernous malformation. ${ }^{12}$ None of these reports utilised MRI to assess the lesions, nor did the lesions involve the supratentorial compartment. This patient provided

a diagnostic and therapeutic challenge. We believe that in this case, surgical resection was necessary for tissue diagnosis and potential adjunctive therapy. Gross total resection also may have provided a potential cure as the patient has remained symptom and recurrence free for over 18 months.

\section{G Rao}

Department of Neurosurgery, University of Utah, 30 N 1900 E, Salt Lake City, Utah 84132, USA

R L Jensen

Department of Neurosurgery, University of Utah, 30 N 1900 E, Salt Lake City, Utah 84132, USA

Correspondence to: Dr Jensen; randy.jensen@hsc.utah.edu

\section{References}

1 Greene GM, Hart MN, Poor MM Jr, et al. Carcinoma metastatic to a cerebellar vascular malformation: case report. Neurosurgery 1990;26:1054-6; discussion 1056-7.

2 Scardigli K, Biller J, Ghobrial M, et al. Brain stem vascular malformation simulating a hemorrhagic metastasis: report of a case with pathologic correlation. Eur Neurol 1984;23:240-6.
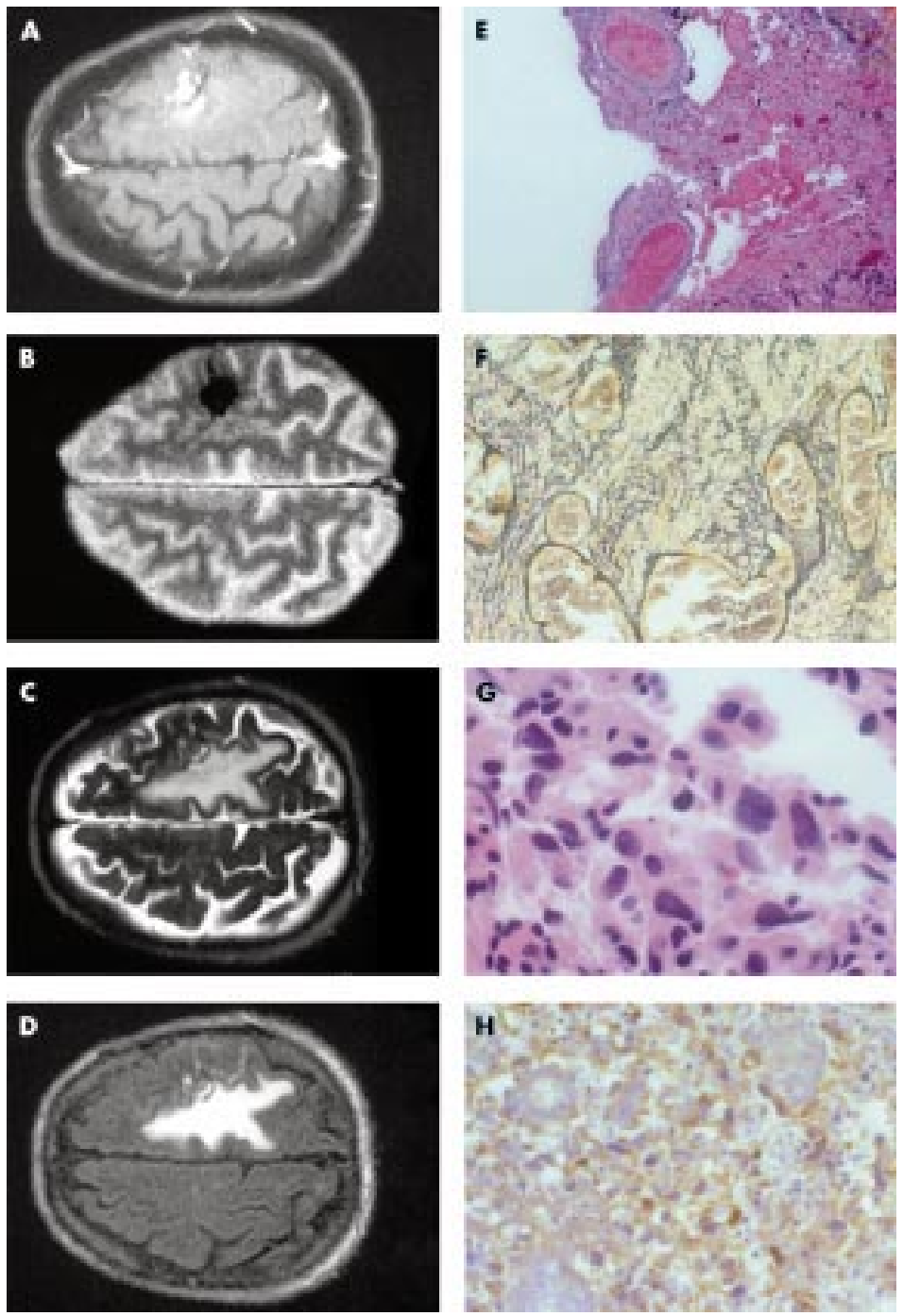

Figure 1 (A) Postcontrast enhanced T1 weighted magnetic resonance imaging (MRI) that shows the left frontal lesion. Note the "popcorn" like appearance, which is consistent with a cavernous malformation. (B) Gradient echo MRI showing the haemorrhagic nature of the lesion. (C) T2-weighted MRI that shows the large amount of oedema associated with the lesion. (D) Fluid attenuated T2 MRI that shows the large amount of oedema. (E) H\&E stain of the surgical specimen revealing numerous loops of vessels consistent with a cavernous malformation. (F) Reticulin stain which reveals the significant endothelial component of the specimen. (G) High-power slide that shows numerous pleomorphic cells with hyperchromatic nuclei and abundant pink cytoplasm consistent with neoplastic cells. (H) Cells stained with AE 1,3 which is specific for keratin. This correlates with an epithelial cancer. 